we were unable to prolong the decalcifying effect of the citric acid until such time as the hæmorrhage cleared up.

CASE 2. The patient was suffering from hæmorrhage into the left Complete decalcification with a dilution of 1 in 1000 of oxalate of ammonium. Two drachms of citric acid were administered three times a day. 15th : Coagulation time, 1 minute 7 seconds. Complete decalcification with a dilution of 1 in 1800 of oxalate of ammonium. 29th : Ooagulation time, 2 minutes. Complete decalcification with a dime, 2 minutes 40 seconds. Complete decalcification with a dilution time, 2 minutes 40 seconds. Complete decalcification with a dilution 55 seconds. Complete decalcification with a dilution 1 in 1050 of oxalate of ammonium. May 10th: Coagulation time, 1 minute 50 seconds. Complete decalcification with a dilution of 1 in 750 of complete decalcification with a dilution of 1 in 1200 of oxalate of ammonium.

CASE 3.-The patient was suffering from hæmorrhage into the left vitreous. March 1st, 1905 : Coagulation time, 50 seconds. Complete Two drachms of citric acid were administered three times a day. 15th. Two drachms of citric acid were administered three times a day. 15th : Coagulation time, 50 seconds. Complete decalcification with a dilution of In 2000 oxalate of ammonium. 29th: Coagulation time, 2 minutes 5 seconds. Complete decalcification with a dilution of 1 in 1400 oxalate of ammonium. April 12th : Coagulation time, 2 minutes 25 seconds.
Complete decalcification with a dilution of 1 in 1500 oxalate of ammonium. 26th: Coagulation time, 2 minutes 5 seconds. Complete decalcification time with a dilution of 1 in 1200 oxalate of ammonium. May 10th: Coagulation time, 1 minute 45 seconds. Complete decalcification with a dilution of 1 in 1050 oxalate of ammonium. 24th : Coagulation time, 55 seconds. Complete decalcification with a dilntion of 1 in 700 oxalate of ammonium.

CASE 4. -The patient was suffering from hæmorrhage into the vitreous. March 15th, 1905: Coagulation time, 1 minute 10 seconds. Complete decalcification with a dilution of 1 in 1500 of oxalate of ammonium. One drachm of citric acid was administered three times a day. 22nd : Coagulation time, 2 minutes 25 seconds. Complete decalcification with a dilution of 1 in 1600 of oxalate of ammonium. April 5th: Coagulation time, 2 minutes 40 seconds. Complete decalcification with a dilution of 1 in 1200 of oxalate of ammonium. 19th : Coagulation time, 2 minutes 10 seconds. Complete decalcification with a dilution of 1 in 1000 of oxalate of ammonium. May 17th Coagulation time, 1 minute 37 seconds. Complete decalcification with a dilution of 1 in 1050 of oxalate of ammonium.

\section{THE USE OF PLASTER-OF-PARIS SPLINTS IN THE TREATMENT OF FRACTURES OF THE LEG.}

By GEORGE WILKINSON, B.A., M.B. CANTAB., F.R.C.S. ENG。,

SURGEON TO THE SHEFFIELD ROYAL HOSPITAL; LECTURER ON PRACTICAL SURGERY IN THE MEDICAL DEPARTMENT OF THE UAIVERSITY OF SHEFFIELD.

THE use of plaster of Paris in the treatment of fractures of the leg is a great convenience to the surgeon, particularly in hospital practice. The pressure on the beds of most general hospitals is often so great that it is necessary to discharge the patients from the wards as soon as it can be done with safety. Once in plaster the fracture is immobilised and may be expected to unite in the course of time in the position in which it was put up. The patient, too, is usually anxious to be released from confinement to bed and to be going about on crutches with his leg in plaster. On the score of convenience we cannot dispense with plaster of Paris in the treatment of these cases unless it be shown that the disadvantages attending its use are serious and unavoidable.

Plaster is used in two forms; as a closed case made with bandages impregnated with plaster and as splints made from some shaped material soaked in plaster cream (Croft's and Bavarian splints). The special disadvantages attaching to the plaster case are that it is difficult to remove and that it loses much of its rigidity when it is " cut up." Removal of the case may be necessitated by swelling of the limb if the plaster has been applied soon after the occurrence of the fracture, or at a later period for shrinkage of the limb and consequent loosening of the case. In either case the application of a fresh plaster will probably be required. To facilitate the cutting up of the case a string may be placed underneath it between the plaster and the flannel bandage surrounding the limb, by means of which a wire or Gigli's saw may be pulled through, with which the case may be divided down the front. Another method is to place a strip of block tin along the front of the leg beneath the plaster, on to which one can cut with a penknife without danger of damaging the patient's skin. In any case, the plaster is apt to be spoiled by being cut up.

Bavarian or Croft's splints get over the difficulty of removal to a certain extent. Their inefficiency in many cases is due to the material of which they are usually made (house flannel soaked in plaster cream). This makes a poor splint, deficient in rigidity, and apt to crumble. Another great disadvantage of the forms of plaster in common use is the uncertainty as to the position of the fragments when the case has been applied. Owing to the manipulation of the limb attending the application of the bandages and the frequent shifting of the hands of the assistant holding the limb it is very easy for the fragments to become displaced and the displacement will only be discovered when it is too late to rectify it-i.e., after the plaster is removed. Another objection to plaster to which much attention has been directed recently is the amount of matting of the soft parts and stiffness of the joints following rigid confinement of the limb in plaster. What is required, therefore, is some form of plaster (1) made of sufficiently rigid and durable material ; (2) applied without shifting the limb about; (3) readily removeable for inspection or treatment by massage and passive movement of the limb ; and (4) which can be opened up without disturbing the fragments or spoiling the case.

I. have been using a form of plaster case for the last two years at the Sheffield Royal Hospital which I consider fulfils all these requirements.

Material.-Gauze such as is used for making gauze bandages is employed. The splints consist of from eight to 12 folds of this gauze, between the layers of which dry plaster is weil rubbed in. Before applying they are immersed in warm water, squeezed out, and then more dry plaster is rubbed into their inner surfaces. No plaster cream should be employed. If the plaster is well baked splints made in this way set very quickly and firmly and are very durable.

Reduction of swelling. - If the limb is much swollen elastic compression is applied to reduce the swelling as a preliminary measure. The limb is wrapped from the toes to above the fracture in layers of non-absorbent wool of a thickness of at least one inch and over this an elastic bandage is applied in simple spirals. The limb is then bandaged on to a back-splint with foot-piece. The swelling: will usually have subsided in three or four days, though in some cases a week is required. The stiffness of the limb subsequently to the removal of the plaster will be much less if care is taken to get rid of the effusion of blood accompanying the fracture in this manner before applying the case.

Blisters. - After being clipped and the serum squeezed out blisters are dressed with two or three layers of lint wrung out of a saturated watery solution of picric acid which dries the surface, preventing any further discharge of serum.

Method of applying the plaster splints.--Supposing the case to be one of fracture of one or both bones of the leg in the lower third, the plaster splints need only be carried as high as the knee. A pattern of the posterior splint is obtained by laying the sound limb on a sheet of paper and cutting round it with scissors. The splint extends from the root of the toes, under the heel, and up the back of the leg to just below the knee. It should be wide enough to embrace about two-thirds of the circumference of the limb. A good width should be allowed at the ankle to accommodate the projection of the heel. The pattern must be turned over so as to reverse the sides in cutting out the splint for the fractured limb (Fig. 1). From 10 to 12 folds of ordinary gauze bandage material should be laid out in a length equal to, and rather wider than, the pattern and cut out to form the posterior splint. This should be laid upon a considerably wider piece of gauze and the whole thickness stitched together down the middle line. Dry plaster should be rubbed in plentifully between the layers of the gauze. The extra width of the outside layer of gauze should now be split into tails on either side of the splint and the tails rolled up so that they may not become "taffled." The posterior splint should now be rolled up from either end on rollers. Two pieces of broomstick answer the purpose. The anterior splint is simply a strip, from three to four inches wide, long enough to reach from the root of the toes to the knee. From eight to ten folds of gauze suffice and the ends should be secured by stitching them together. Plaster is rubbed in as in the case of the posterior splint and the splint is rolled up on a piece of broomstick. The leg should then be bandaged evenly with a single thickness of flannel, a pad of wool being placed on either side above the heel, this being a point on which the splint is likely to cause pressure. The knee should be bent to a right angle and the leg laid upon a support, such 
as a deal box. I have had a box made, of the form shown in Fig. 2, which elevates the limb 19, 17, or 15 inches, according to which side is turned uppermost. Both anterior and posterior splints on their rollers are now placed in warm water for a few minutes to moisten the plaster. The surgeon then makes traction from the heel and foot. Counter traction is obtained by passing a clove hitch round the upper part of the thigh and tying

FIG. 1.

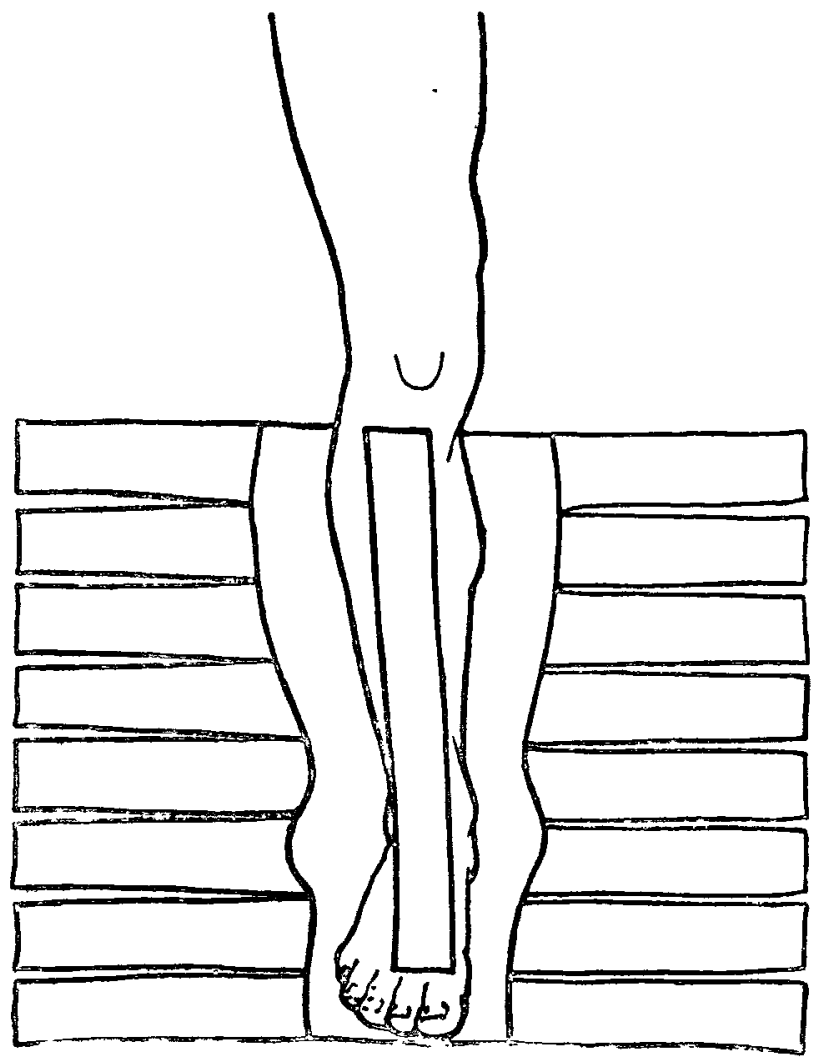

Showing position of leg and of splint before application.

to the head of the bed. Flexing the knee to a right angle eliminates one of the chief obstacles to reduction of the fragments by relaxing the gastrocneu ius muscle. If the foot is kept at right angles to the leg and the inner side of the great toe in a line with the internal malleolus and the inner side of the patella the fragments will come into correct position on making traction. The

FIG. 2.

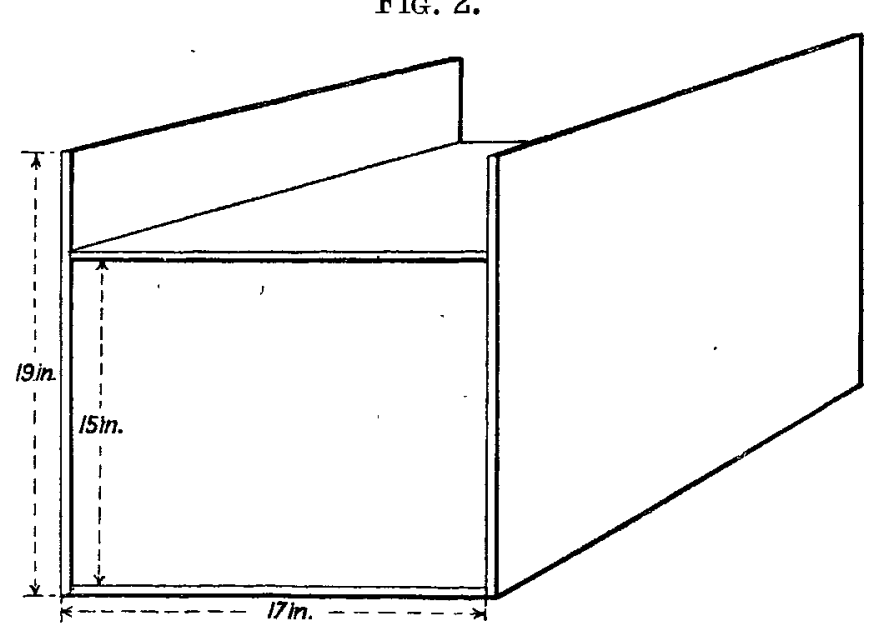

Box used to support the leg with the knee flexed to a right angle.

anterior splint is now unrolled and laid along the front of the leg and foot. The posterior splint is removed from the water and well squeezed out. It is then unrolled and laid under the limb. (Fig. 3.) Some additional plaster may be rubbed into its upper surface. Whilst the surgeon keeps up traction and maintains the foot in the correct position an assistant brings the posterior splint round the under surface of the leg and foot and fixes it in position by tying the tails oyer the anterior splint. It is better to avoid tying the knots directly over the sharp anterior margin of the shin-bone, where they may cause undue pressure. Traction with the foot in the correct position must be kept up until the plaster is set sufficiently hard to prevent displacement of the fragments ; three minutes generally suffice. The leg is left for about half an hour lying flexed on the box with a support, such as a thin book, under the heel and hot-water bottles or bricks on either side to hasten the drying of the plaster. After about 12 hours the plaster will be perfectly hard and the patient may then be allowed up on crutches. Unless there is any special reason for cutting up the case it is usually left untouched for about three weeks, when a fair amount of union may be expected to have taken place. It is then cut up with a pair of scissors along the interval between the two splints and subsequently the limb should be removed from the case about twice a week for passive movements of the ankle. After replacing it the splints should be bandaged firmly with a calico roller.

If the fracture is above the lower third of the leg it will be necessary to fix the knee-joint. The splints may be put on in the same way, except that the knee will have to be in the extended position and the splints carried as high as the middle of the thigh.

In fractures the result of great violence, where there is comminution or much damage to the soft parts, rest in bed for a time is essential. Even in this class of case plaster

\section{FIG. 3.}

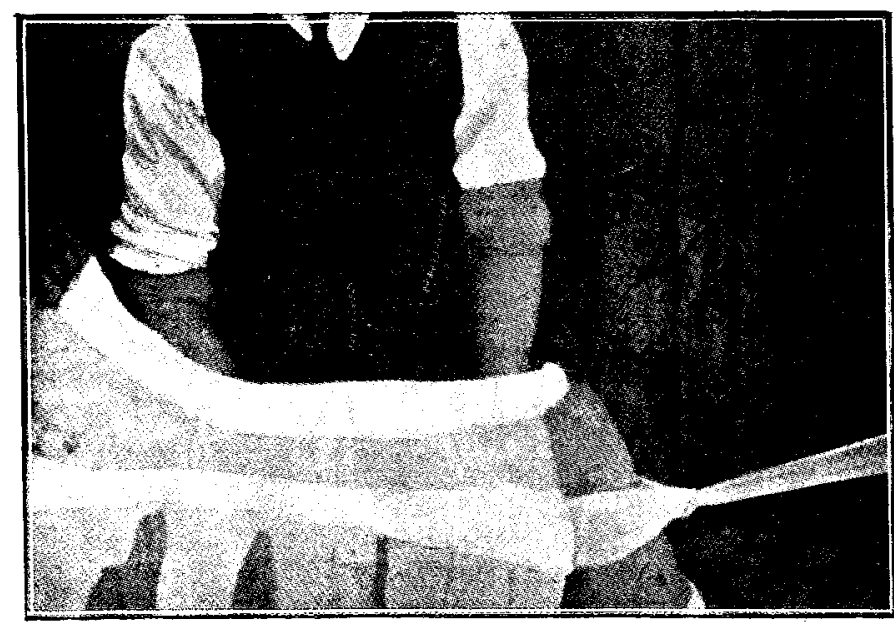

Fracture of both bones of the leg in the lou er third. Anterior and posterior splints in position; knee flexed to a right angle.

splints applied in a similar manner offer distinct advantages in the way of complete immobilisation and accurate retention of fragments in position after reduction, a result often difficult to obtain with any other form of splint, whilst the readiness with which the case can be opened up without disturbing the leg in any way allows the condition of the limb to be kept under as close observation as when any other form of splint is used. In this class of case the limb is put up flexed at an angle of from 135 to 150 degrees and the splints should reach as high as the upper third of the thigh. In applying the splints a pad of wool should be placed next the skin of the under surface of the thigh just above the knee-joint, where the pressure of the assistant's hand in making counter extension makes a dent in the plaster, which may chafe the skin unless protected by a pad. Less severe cases of compound fracture may be treated in the same way after thorough cleansing and dressing of the wound, the portion of the case in the neighbourhood of the wound being lined with a piece of thin mackintosh. The only class of case for which the plaster splints are unsuited is severe compound fractures where there is likely to be much discharge or where it may be necessary to employ irrigation.

In the case of oblique fractures, where reduction of the displacement is often difficult and retention of the fragments in the reduced position is more difficult still, the posterior plaster splint answers well. In these cases the anterior splint should be dispensed with, so that the position of the bones during traction on the limb may be observed. When once the displacement is reduced the plaster splint immobilises the fragments more perfectly than any other apparatus. As soon as the plaster is hard the "tails" with which it is tied 
on should be cut away and a firm 'flannel bandage should be applied in place of them, the limb in its case being subsequently fixed on to a Neville's back splint and swung from a cradle. When union is fairly firm an ordinary straight plaster may be substituted for the suspension splint and the patient allowed up on crutches.

Pott's fracture.-One of the most useful applications of this form of plaster case is in the treatment of Pott's fracture. Preliminary treatment by elastic pressure made over layers of cotton-wool is required in most cases, it being particularly desirable to get rid of the effusion in and around the anklejoint. The pattern for the posterior splint is cut to embrace more of the inner than of the outer side of the leg and foot, so as to leave the fibula exposed. The knee should be bent to a right angle and the leg laid on a box. When this is done there is, as a rule, no difficulty whatever in reducing the displacement. Counter traction may be made by an assistant at the knee, or by passing a bandage round the lower part of the thigh just above the knee, and tying it to the head of the bed. An assistant will be required to hold the leg steady whilst the surgeon manipulates the foot, and another to apply the splints by tying the "tails" of the outside bandage. The splints are first fixed around the leg above the ankle and the surgeon then strongly adducts and inverts the foot, keeping it at the same time at right angles to the leg and pulling it forwards. In this way the outward and backward displacement is corrected. The splints are then fixed round the foot and ankle and the surgeon holds the foot in the correct position until the plaster has set. The ready removeability of the splints is a great advantage in the after-treatment of these cases. I begin passive movement of the ankle at the end of three weeks. Systematic massage to the ankle and leg muscles is most useful. Owing to the number of fracture cases occurring in hospital practice it is practically impossible to employ massage in more than a small proportion of them.

To recapitulate the advantages claimed for this form of plaster case. 1. It is applicable to all cases of fracture of the leg, with the exception of certain cases of compound fracture. 2. Its method of application is simple. 3. It is rigid and durable. 4. It can readily be cut up without disturbing the limb, and can be reapplied without loss of rigidity. 5 . It is put on with the limb in the most favourable position for reduction of the displacement-i.e., with the knee flexed and the gastrocnemius relaxed. 6. The position of the leg is not shifted and traction on the axis of the limb is kept up during the whole time of application of the splints. Consequently there is a fair certainty of the fragments being fixed in good position. On the other hand, it must be admitted that the limb does not present quite so neat an appearance when put up in this manner as it does in a well made plaster case applied in the ordinary way. I believe that what is lost in neatness is gained in efficiency.

Sheffield.

\section{A CASE OF DEMENTIA DUE TO HUNTINGDON'S CHOREA.}

BY G. E. PEACHELL, M.B., B.S. LOND., M.R.C.S. ENG., L.R.C.P. LOND.

ASSISTANT MEDICAL OFFICER, WEST SUSSEX COUNTY ASYLUMM.

THE following case may prove of some interest as the condition is somewhat rare and it is seldom that a necropsy is obtainable, though this failed to throw much light on the pathology of this obscure disease.

A married man, aged 54 years, was admitted into the West Sussex County Asylum on Feb. 18th, 1905, suffering from dementia due to Huntingdon's chorea. His family history was as follows:-

Grandmother :

had disease badly and died from it.

$$
\text { , and died fro }
$$

\begin{tabular}{|c|c|c|c|c|}
\hline $\begin{array}{r}\text { Mother affecter } \\
\text { age about } 56 \\
\text { who d } \\
\end{array}$ & $\begin{array}{l}\text { d ; died from the } \\
\text { years; married } \\
\text { ied from cancer. } \\
\text { I }\end{array}$ & disease; & $\begin{array}{c}\text { Son } \\
\text { (healthy). }\end{array}$ & Son \\
\hline $\begin{array}{l}\text { The patient; } \\
\text { arried } 25 \text { years; } \\
\text { no issue. }\end{array}$ & $\begin{array}{c}\text { Brother } \\
\text { (whereabouts } \\
\text { unknown). }\end{array}$ & $\begin{array}{r}\text { Sister aft } \\
\text { chiefly f } \\
\text { prese }\end{array}$ & $\begin{array}{l}\text { eected; } \\
\text { ace at } \\
\text { int. }\end{array}$ & $\begin{array}{l}\text { Sister } \\
\text { has Bright's } \\
\text { disease). }\end{array}$ \\
\hline
\end{tabular}

The disease started in 1896 when the patient was 45 years old. Previously to this he had never been ill. As is usually the case, the irregular convulsive movements were first noticed in the face, then gradually involved the neck and shoulder muscles and spread to the upper and lower extremities, and quite lately the muscles of the tongue and of deglutition became affected. He had been unable to follow his occupation for the last six years and the disease had rapidly progressed during the last six months; recently he had got worse mentally and had become very irritable and impulsive and a source of danger to children on the road. The patient was a tall, fairly wellnourished man; he walked with a swaying gait from the hips, keeping the legs rigid. The whole body, but more especially the left side of the face, arm, and leg, underwent coarse, irregular, incoördinate movements, which be could not control but which ceased temporarily if he put his muscles into action, such as by grasping both his hands; the movements were absent altogether during sleep. The tongue and lip muscles were markedly affected and he had slight difficulty in swallowing. His eyes were prominent; bis pupils were equal and active. He could not now write his name but made jerky, irregular strokes when he attempted to do so, though he could hold the pen steadily when not using it. Sensation and the reflexes appeared to be normal and he bad command over his bladder and rectum. There was no evidence of disease in the heart, lungs, or abdomen. The patient was dull and slow in answering questions but had much difficulty in articulation and so was hard to follow. His mental faculties were much impaired and his memory was very poor. He complained of headache and pain over the left side of the head and face and was constantly putting his hand up and rubbing his forehead. He was put on a mince diet and an occasional dose of chloral at night. $\mathrm{He}$ soon had to take to fluids as he could not swallow mince. He gradually got worse, became very sluggish mentally, and at times irritable and impulsive, and died rather suddenly on March 13th.

Necropsy.-At the post-mortem examination, held 16 hours after death, the skull-cap was somewhat thickened; the dura mater was normal. The pia arachnoid was much thickened and had an opaque gelatinous appearance ; there was a good deal of fluid between the membranes and the cortex, allowing them to strip readily from the convolutions. There was much cerebral atrophy which was especially marked in the frontal lobes. There was no excess of fluid in the ventricles, but a fine granular condition of the ependyma of the lateral ventricles and lateral recesses of the fourth ventricle was present. The cerebral vessels were engorged the basal vessels were healthy. The cord looked healthy to the naked eye. The pons, medulla, and cerebellum showed no pathological change. Except that both lower lobes of the lungs were congested the rest of the organs were healthy. Microscopical sections of the cortex of the ascending parietal and ascending frontal convolutions and from the frontal lobes on both sides, and sections of the cord-cervical, dorsal, and lumbar-hardened in Müller's fluid and stained by van Gieson's method, failed to show any marked pathologica] change.

With regard to diagnosis and prognosis little need be said. The diagnosis is readily made in the vast number of cases from (1) the hereditary nature of the malady, occurring in successive generations ; (2) the character of the involuntary movements; (3) the chronicity of the complaint; and (4) the mental deterioration associated with it. It may, however, be confounded with Friedreich's ataxy, symptomatic hemi-chorea, possibly with some cases of locomotur ataxy, and in the early stages, when the face is alone affected, with convulsive tic. The prognosis is always grave, recovery is unknown, and though the pitiable sufferer may live to an advanced age he is rendered incapable of supporting himself by reason of the restless movements and progressive mental enfeeblement. No treatment is of any avail in arresting the disease, though potassium bromide, chloral, and antipyrin are occasionally useful in quieting the patient and obtaining sleep. Hydrotherapy appears to be useless.

The interest lies in the pathology of the disease. Dr. Huet in his masterly monograph "De la Chorée Chronique" remarks that so many different lesions have been found post mortem that it is impossible to judge of their respective value; he puts on one side the "embolic theory" as quite unproven, and suggests that the lesion lies in some change in the cortical nerve cells which are inherently prone to undergo degeneration at a prematurely early age ; comparing the disease with epilepsy it seems likely that such may prove 